GLASNIK MATEMATIČKI

Vol. 55(75)(2020), $177-190$

\title{
ON SOME PARTIAL ORDERS ON A CERTAIN SUBSET OF THE POWER SET OF RINGS
}

\author{
Gregor Dolinar, Bojan Kuzma, Janko Marovt and Burcu Ungor \\ University of Ljubljana, University of Primorska, University of Maribor, \\ Slovenia and Ankara University, Turkey
}

\begin{abstract}
Let $\mathcal{R}$ be a ring with identity and let $\mathcal{J}_{\mathcal{R}}$ be a collection of subsets of $\mathcal{R}$ such that their left and right annihilators are generated by the same idempotent. We extend the notion of the sharp, the left-sharp, and the right-sharp partial orders to $\mathcal{J}_{\mathcal{R}}$, present equivalent definitions of these orders, and study their properties. We also extend the concept of the core and the dual core orders to $\mathcal{J}_{\mathcal{R}}$, show that they are indeed partial orders when $\mathcal{R}$ is a Baer *-ring, and connect them with one-sided sharp and star partial orders.
\end{abstract}

\section{INTRODUCTION}

Let $\mathcal{S}$ be a semigroup and $a \in \mathcal{S}$. We say that $a$ has an inner generalized inverse $a^{-} \in \mathcal{S}$ if $a=a a^{-} a$, and a reflexive generalized inverse $a^{\prime} \in \mathcal{S}$ if $a=$ $a a^{\prime} a, a^{\prime}=a^{\prime} a a^{\prime}$. A semigroup in which every element has an inner generalized inverse is called a regular semigroup. A reflexive generalized inverse of $a$ that commutes with $a$ is called the group inverse of $a$. The group inverse, which is unique if it exists (see [10]), is denoted by $a^{\sharp}$.

Let $\mathcal{R}$ be a ring and let us denote by $\mathcal{G}(\mathcal{R})$ the subset of elements in $\mathcal{R}$ which have the group inverse. Let $M_{n}(\mathbb{F})$ be the ring of all $n \times n$ matrices over a field $\mathbb{F}$. In [18] (see also [19]), Mitra introduced the sharp partial order on

2020 Mathematics Subject Classification. 06F25, 06A06, 15A09.

Key words and phrases. Baer *-ring, sharp partial order, core partial order, star partial order, one-sided partial order.

The authors acknowledge the financial support from the Slovenian Research Agency, ARRS (research core funding No. P1-0222, No. P1-0285, and No. P1-0288, and SloveneTurkish Grant BI-TR/17-19-004) and by the Scientific and Technological Research Council of Turkey, TUBITAK (Grant TUBITAK-116F435). The authors wish to thank ARRS and TUBITAK for financial support. 
$\mathcal{G}\left(M_{n}(\mathbb{F})\right)$. This order was generalized in $[15]$ to $\mathcal{G}(\mathcal{R})$ where $\mathcal{R}$ is an arbitrary ring with identity: For $a, b \in \mathcal{G}(\mathcal{R})$ we write

$$
a \leq^{\sharp} b \quad \text { if } \quad a^{\sharp} a=a^{\sharp} b \text { and } a a^{\sharp}=b a^{\sharp} \text {. }
$$

In [15], it was shown that $\leq^{\sharp}$ is indeed a partial order on $\mathcal{G}(\mathcal{R})$. Recently an application of this order to autonomous linear systems has been presented in [11]. In particular, if real $n \times n$ state matrices of two autonomous linear systems are related under the sharp partial order, then the difference of their solutions is again a solution of another autonomous linear system. The minus partial order $\leq^{-}$is another well-known partial order on a semigroup whose elements have reflexive generalized inverses. This order was originally defined by Hartwig in [9]. For a regular semigroup $\mathcal{S}$ and $a, b \in \mathcal{S}$ we write

$$
a \leq^{-} b \text { if } a^{\prime} a=a^{\prime} b \text { and } a a^{\prime}=b a^{\prime}
$$

where $a^{\prime}$ is a reflexive generalized inverse of $a$. It turns out that $a \leq^{-} b$ if and only if $a^{-} a=a^{-} b$ and $a a^{-}=b a^{-}$for some inner generalized inverse $a^{-}$of $a$. We remark that the minus partial order was one of the first orders which was generalized and extensively studied in the literature. Let us present a brief summary of some results on this order. This also motivates the main definitions (see Definitions 2.2-4.4) of the sharp and related partial orders which is the main topic of our study. The main idea is to describe the orders in terms of the annihilators.

The algebra $B(\mathcal{H})$ of all bounded linear operators on a Hilbert space $\mathcal{H}$ is an example of a semigroup that is not necessarily regular. Namely, $A \in B(\mathcal{H})$ is regular if and only if the image of $A$ is closed (see for example [21]). It follows that if $\mathcal{H}$ is finite dimensional, then $B(\mathcal{H})$ is a regular semigroup. Let $\operatorname{Ker} A, \operatorname{Im} A, \overline{\operatorname{Im} A}$ denote the kernel, the image and the closure of the image of $A \in B(\mathcal{H})$, respectively. Motivated by Hartwig's definition of the minus partial order (1.2), Šemrl introduced in [23] a new order $\leq_{S}$ on $B(\mathcal{H})$ in the following way: For $A, B \in B(\mathcal{H})$ we write $A \leq_{S} B$ if there exist idempotent operators $P, Q \in B(\mathcal{H})$ such that $\operatorname{Im} P=\overline{\operatorname{Im} A}$, Ker $A=\operatorname{Ker} Q, P A=P B$, and $A Q=B Q$. Šemrl called this order the minus partial order on $B(\mathcal{H})$ and proved that this is indeed a partial order on $B(\mathcal{H})$ for a general Hilbert space $\mathcal{H}$. He also showed that the partial order $\leq_{S}$ is the same as Hartwig's minus partial order $\leq^{-}$when $\mathcal{H}$ is finite dimensional.

For a subset $\mathcal{A}$ of a ring $\mathcal{R}$, let $l_{\mathcal{R}}(\mathcal{A})=\{x \in \mathcal{R}: x a=0$ for every $a \in \mathcal{A}\}$ and $r_{\mathcal{R}}(\mathcal{A})=\{x \in \mathcal{R}: a x=0$ for every $a \in \mathcal{A}\}$ stand for the left annihilator and the right annihilator of $\mathcal{A}$ in $\mathcal{R}$, respectively. If the subset $\mathcal{A}$ is a singleton, say $\mathcal{A}=\{a\}$, then we simply write $l_{\mathcal{R}}(a)$ and $r_{\mathcal{R}}(a)$, respectively. A ring $\mathcal{R}$ is called a Rickart ring if for every $a \in \mathcal{A}$ there exist idempotent elements $p, q \in \mathcal{A}$ such that $r_{\mathcal{R}}(a)=p \mathcal{A}$ and $l_{\mathcal{R}}(a)=\mathcal{A} q$. Note that every Rickart ring $\mathcal{A}$ has the (multiplicative) identity (see [4] or [12]). An example of a Rickart ring is the algebra $B(\mathcal{H})$. Observe that for $A, B \in B(\mathcal{H})$ we have (see $[17$, 
Lemma 2.1])

$$
r_{B(\mathcal{H})}(A)=r_{B(\mathcal{H})}(B) \text { if and only if } \operatorname{Ker} A=\operatorname{Ker} B
$$

and

$$
l_{B(\mathcal{H})}(A)=l_{B(\mathcal{H})}(B) \quad \text { if and only if } \overline{\operatorname{Im} B}=\overline{\operatorname{Im} A} .
$$

Unless stated otherwise, let $\mathcal{R}$ denote a ring with the multiplicative identity 1 from now on. Note that for an idempotent $p \in \mathcal{R}, l_{\mathcal{R}}(p)=\mathcal{R}(1-p)$ and $r_{\mathcal{R}}(p)=(1-p) \mathcal{R}$ (see [5, Lemma 2.1]). These observations lead to the following generalization of the minus order ([5, Definition 2.1]). For $a, b \in \mathcal{R}$ we write $a \leq^{-} b$ if there exist idempotent elements $p, q \in \mathcal{R}$ such that $l_{\mathcal{R}}(a)=\mathcal{R}(1-p), r_{\mathcal{R}}(a)=(1-q) \mathcal{R}, p a=p b$, and $a q=b q$. It was shown in [5] that this is indeed a partial order when $\mathcal{R}$ is a Rickart ring.

In [12], Baer rings were introduced as rings in which the right annihilator of every subset is the principal right ideal generated by an idempotent. In a Baer ring, left annihilators are also idempotent-generated, so clearly, every Baer ring is a Rickart ring. A ring equipped with an involution $*$ is called a $*$-ring. A $*$-ring $\mathcal{R}$ is said to be a Baer $*$-ring if for every subset $\mathcal{A}$ of $\mathcal{R}$, the right (equivalently, left) annihilator of $\mathcal{A}$ is the principal right (left) ideal generated by a projection (i.e. a self-adjoint idempotent). In [24], the notion of the minus partial order was extended to the power sets of Baer rings. We denote the power set of a ring $\mathcal{R}$ by $\mathcal{P}(\mathcal{R})$. Let $\mathcal{R}$ be a ring and $\mathcal{A}, \mathcal{B} \subseteq \mathcal{R}$. We write

$$
\mathcal{A} \leq^{-} \mathcal{B} \quad \text { if } \quad l_{\mathcal{R}}(\mathcal{A})=l_{\mathcal{R}}(p), r_{\mathcal{R}}(\mathcal{A})=r_{\mathcal{R}}(q), p \mathcal{A}=p \mathcal{B}, \mathcal{A} q=\mathcal{B} q
$$

for some idempotent elements $p, q \in \mathcal{R}$. We call the relation $\leq^{-}$the minus relation on $\mathcal{P}(\mathcal{R})$.

In [24], it was proved that when $\mathcal{R}$ is a Baer ring, the relation introduced with (1.3) is a partial order on $\mathcal{P}(\mathcal{R})$.

For a ring $\mathcal{R}$ with identity, let

$$
\mathcal{I}_{\mathcal{R}}=\left\{a \in \mathcal{R}: r_{\mathcal{R}}(a)=r_{\mathcal{R}}(p) \text { and } l_{\mathcal{R}}(a)=l_{\mathcal{R}}(p)\right.
$$

for some idempotent $p \in \mathcal{R}\}$.

It turns out (see [22]) that when $\mathcal{R}=B(\mathcal{H})$ with $\operatorname{dim} \mathcal{H}<\infty, \mathcal{I}_{\mathcal{R}}$ is exactly the set $\mathcal{G}(\mathcal{R})$ of all group invertible operators in $B(\mathcal{H})$. Independently to [15] where definition (1.1) was introduced, Rakić presented in [22] another generalization of the sharp partial order to rings using annihilators. Namely, for $a, b \in \mathcal{R}$, we say that $a \leq^{\sharp} b$ if $a \in \mathcal{I}_{\mathcal{R}}$ and $a=p b=b p$ for some idempotent $p \in \mathcal{R}$ with $r_{\mathcal{R}}(a)=r_{\mathcal{R}}(p)$ and $l_{\mathcal{R}}(a)=l_{\mathcal{R}}(p)$.

Let now

$$
\begin{gathered}
\mathcal{J}_{\mathcal{R}}=\left\{\mathcal{A} \subseteq \mathcal{R}: r_{\mathcal{R}}(\mathcal{A})=r_{\mathcal{R}}(p) \text { and } l_{\mathcal{R}}(\mathcal{A})=l_{\mathcal{R}}(p)\right. \\
\text { for some idempotent } p \in \mathcal{R}\} .
\end{gathered}
$$


The first goal of this paper is to extend the concept of the sharp partial order $\leq \#$ (introduced on $\mathcal{I}_{\mathcal{R}}$ by Rakić in [22]) to $\mathcal{J}_{\mathcal{R}}$ and study its properties. This is done in Section 2. In Section 3 we present the concept of the left-sharp and the right-sharp partial orders on $\mathcal{J}_{\mathcal{R}}$, and finally, in Section 4 , we introduce the core and the dual core partial orders to $\mathcal{J}_{\mathcal{R}}$ and show that they are indeed partial orders when the ring $\mathcal{R}$ is a Baer $*$-ring.

\section{THE SHARP ORDER}

Suppose $\mathcal{A} \in \mathcal{J}_{\mathcal{R}}$ and let $p, q \in \mathcal{R}$ be two idempotents such that $r_{\mathcal{R}}(\mathcal{A})=$ $r_{\mathcal{R}}(p), l_{\mathcal{R}}(\mathcal{A})=l_{\mathcal{R}}(p)$, and $r_{\mathcal{R}}(\mathcal{A})=r_{\mathcal{R}}(q), l_{\mathcal{R}}(\mathcal{A})=l_{\mathcal{R}}(q)$. It follows that $l_{\mathcal{R}}(p)=l_{\mathcal{R}}(q)$ and $r_{\mathcal{R}}(p)=r_{\mathcal{R}}(q)$, and therefore $(1-p) q=0=q(1-p)$, i.e. $q=p q=q p$, and $(1-q) p=0=p(1-q)$, i.e. $p=q p=p q$. We may conclude that the idempotent $p \in \mathcal{R}$, such that $r_{\mathcal{R}}(\mathcal{A})=r_{\mathcal{R}}(p)$ and $l_{\mathcal{R}}(\mathcal{A})=l_{\mathcal{R}}(p)$ for $\mathcal{A} \in \mathcal{J}_{\mathcal{R}}$, is unique. We denote it by $p_{\mathcal{A}}$.

REMARK 2.1. Let $\mathcal{A} \in \mathcal{J}_{\mathcal{R}}$. Since then $r_{\mathcal{R}}(\mathcal{A})=r_{\mathcal{R}}\left(p_{\mathcal{A}}\right)$ and $l_{\mathcal{R}}(\mathcal{A})=$ $l_{\mathcal{R}}\left(p_{\mathcal{A}}\right)$, it follows that $\left(1-p_{\mathcal{A}}\right) \mathcal{A}=0=\mathcal{A}\left(1-p_{\mathcal{A}}\right)$ and thus

$$
\mathcal{A}=p_{\mathcal{A}} \mathcal{A}=\mathcal{A} p_{\mathcal{A}} .
$$

We now introduce the notion of the sharp order on $\mathcal{J}_{\mathcal{R}}$.

Definition 2.2. Let $\mathcal{A} \in \mathcal{J}_{\mathcal{R}}$ and $\mathcal{B} \subseteq \mathcal{R}$. We write $\mathcal{A} \leq \sharp \mathcal{B}$ when $p_{\mathcal{A}} \mathcal{A}=p_{\mathcal{A}} \mathcal{B}$ and $\mathcal{A} p_{\mathcal{A}}=\mathcal{B}_{\mathcal{A}_{\mathcal{A}}}$. We call the relation $\leq \sharp$ the sharp order on $\mathcal{J}_{\mathcal{R}}$.

Next, we show that the relation $\leq \sharp$ is a partial order for any ring $\mathcal{R}$ with identity. First, let us present an auxiliary result.

LEMma 2.3. Let $\mathcal{A} \in \mathcal{J}_{\mathcal{R}}, \mathcal{B} \subseteq \mathcal{R}$, and $\mathcal{A} \leq \sharp \mathcal{B}$. Then $r_{\mathcal{R}}(\mathcal{B}) \subseteq r_{\mathcal{R}}(\mathcal{A})$ and $l_{\mathcal{R}}(\mathcal{B}) \subseteq l_{\mathcal{R}}(\mathcal{A})$.

Proof. Let $\mathcal{A} \leq \sharp \mathcal{B}$. Then $p_{\mathcal{A}} \mathcal{A}=p_{\mathcal{A}} \mathcal{B}$, and $\mathcal{A} p_{\mathcal{A}}=\mathcal{B} p_{\mathcal{A}}$. By Remark $2.1, \mathcal{A}=p_{\mathcal{A}} \mathcal{B}=\mathcal{B}_{p_{\mathcal{A}}}$. Suppose $d \in r_{\mathcal{R}}(\mathcal{B})$. Then $\mathcal{B} d=0$ and thus $p_{\mathcal{A}} \mathcal{B} d=0$. Hence, $\mathcal{A} d=0$, i.e. $d \in r_{\mathcal{R}}(\mathcal{A})$. So, $r_{\mathcal{R}}(\mathcal{B}) \subseteq r_{\mathcal{R}}(\mathcal{A})$. We similarly show that $l_{\mathcal{R}}(\mathcal{B}) \subseteq l_{\mathcal{R}}(\mathcal{A})$

THEOREM 2.4. The relation $\leq \sharp$ introduced with Definition 2.2 is a partial order on $\mathcal{J}_{\mathcal{R}}$.

Proof. Reflexivity: Clearly, $\mathcal{A} \leq \sharp \mathcal{A}$ for every $\mathcal{A} \in \mathcal{J}_{\mathcal{R}}$.

Antisymmetry: Let $\mathcal{A}, \mathcal{B} \in \mathcal{J}_{\mathcal{R}}$ with $\mathcal{A} \leq \sharp \mathcal{B}$ and $\mathcal{B} \leq \sharp \mathcal{A}$. Then $p_{\mathcal{A}} \mathcal{A}=$ $p_{\mathcal{A}} \mathcal{B}, \mathcal{A} p_{\mathcal{A}}=\mathcal{B} p_{\mathcal{A}}$, and $p_{\mathcal{B}} \mathcal{B}=p_{\mathcal{B}} \mathcal{A}, \mathcal{B}_{p_{\mathcal{B}}}=\mathcal{A} p_{\mathcal{B}}$. Since then $\mathcal{A}=p_{\mathcal{A}} \mathcal{B}=\mathcal{B} p_{\mathcal{A}}$ and $\mathcal{B}=p_{\mathcal{B}} \mathcal{B}=\mathcal{B} p_{\mathcal{B}}=p_{\mathcal{B}} \mathcal{A}=\mathcal{A} p_{\mathcal{B}}$, it follows that $\mathcal{A}=p_{\mathcal{A}} \mathcal{B}=p_{\mathcal{A}} \mathcal{B} p_{\mathcal{B}}=$ $\mathcal{A} p_{\mathcal{B}}=\mathcal{B}$.

Transitivity: Let $\mathcal{A}, \mathcal{B} \in \mathcal{J}_{\mathcal{R}}$ and $\mathcal{C} \subseteq \mathcal{R}$ with $\mathcal{A} \leq \sharp \mathcal{B}$ and $\mathcal{B} \leq \sharp \mathcal{C}$. Then $p_{\mathcal{A}} \mathcal{A}=p_{\mathcal{A}} \mathcal{B}, \mathcal{A} p_{\mathcal{A}}=\mathcal{B} p_{\mathcal{A}}$, and $p_{\mathcal{B}} \mathcal{B}=p_{\mathcal{B}} \mathcal{C}, \mathcal{B} p_{\mathcal{B}}=\mathcal{C}_{p_{\mathcal{B}}}$. By Lemma 2.3, we have $r_{\mathcal{R}}\left(p_{\mathcal{B}}\right)=r_{\mathcal{R}}(\mathcal{B}) \subseteq r_{\mathcal{R}}(\mathcal{A})=r_{\mathcal{R}}\left(p_{\mathcal{A}}\right)$ and $l_{\mathcal{R}}\left(p_{\mathcal{B}}\right)=l_{\mathcal{R}}(\mathcal{B}) \subseteq l_{\mathcal{R}}(\mathcal{A})=$ 
PARTIAL ORDERS ON A CERTAIN SUBSET OF THE POWER SET OF RINGS 181

$l_{\mathcal{R}}\left(p_{\mathcal{A}}\right)$. So, $p_{\mathcal{A}}\left(1-p_{\mathcal{B}}\right)=0=\left(1-p_{\mathcal{B}}\right) p_{\mathcal{A}}$ and thus $p_{\mathcal{A}}=p_{\mathcal{A}} p_{\mathcal{B}}=p_{\mathcal{B}} p_{\mathcal{A}}$. From $\mathcal{B} p_{\mathcal{B}}=\mathcal{C} p_{\mathcal{B}}$ we obtain $\mathcal{B} p_{\mathcal{B}} p_{\mathcal{A}}=\mathcal{C} p_{\mathcal{B}} p_{\mathcal{A}}$ and thus $\mathcal{A} p_{\mathcal{A}}=\mathcal{B} p_{\mathcal{A}}=\mathcal{C} p_{\mathcal{A}}$. Similarly, $p_{\mathcal{B}} \mathcal{B}=p_{\mathcal{B}} \mathcal{C}$ yields $p_{\mathcal{A}} \mathcal{A}=p_{\mathcal{A}} \mathcal{B}=p_{\mathcal{A}} \mathcal{C}$. It follows that $\mathcal{A} \leq \sharp \mathcal{C}$.

With the next result we present new characterizations (i.e. equivalent definitions) of the sharp partial order on $\mathcal{J}_{\mathcal{R}}$.

Theorem 2.5. Let $\mathcal{A} \in \mathcal{J}_{\mathcal{R}}$ and $\mathcal{B} \subseteq \mathcal{R}$. Then the following statements are equivalent.

(i) $\mathcal{A} \leq^{\sharp} \mathcal{B}$.

(ii) There exists an idempotent $p \in \mathcal{R}$ such that $\mathcal{A}=p \mathcal{A} p=p \mathcal{B} p, p \mathcal{B}(1-$ $p)=\{0\}$, and $(1-p) \mathcal{B} p=\{0\}$.

(iii) There exists an idempotent $p \in \mathcal{R}$ such that $l_{\mathcal{R}}(p) \subseteq l_{\mathcal{R}}(\mathcal{A}), r_{\mathcal{R}}(p) \subseteq$ $r_{\mathcal{R}}(\mathcal{A}), p \mathcal{A}=p \mathcal{B}$, and $\mathcal{A} p=\mathcal{B} p$.

(iv) There exists an idempotent $p \in \mathcal{R}$ such that $\mathcal{A}=p \mathcal{B}=\mathcal{B} p$.

Proof. (i) $\Rightarrow$ (ii): Trivial.

(ii) $\Rightarrow$ (iii): Suppose there exists an idempotent $p \in \mathcal{R}$ such that $\mathcal{A}=$ $p \mathcal{A} p=p \mathcal{B} p, p \mathcal{B}(1-p)=\{0\}$, and $(1-p) \mathcal{B} p=\{0\}$. Then $p \mathcal{B}=p \mathcal{B} p$ and $\mathcal{B} p=p \mathcal{B} p$ and therefore

$$
p \mathcal{A}=\mathcal{A}=\mathcal{A} p=p \mathcal{B} p=p \mathcal{B}=\mathcal{B} p .
$$

Also, $(1-p) \mathcal{A}=\{0\}=\mathcal{A}(1-p)$ and therefore $l_{\mathcal{R}}(p) \subseteq l_{\mathcal{R}}(\mathcal{A})$ and $r_{\mathcal{R}}(p) \subseteq$ $r_{\mathcal{R}}(\mathcal{A})$.

(iii) $\Rightarrow\left(\right.$ i): Suppose (iii) holds. Since $l_{\mathcal{R}}(p) \subseteq l_{\mathcal{R}}(\mathcal{A}), r_{\mathcal{R}}(p) \subseteq r_{\mathcal{R}}(\mathcal{A})$, we have $l_{\mathcal{R}}(p) \subseteq l_{\mathcal{R}}\left(p_{\mathcal{A}}\right), r_{\mathcal{R}}(p) \subseteq r_{\mathcal{R}}\left(p_{\mathcal{A}}\right)$, and thus $p_{\mathcal{A}}(1-p)=0=(1-p) p_{\mathcal{A}}$, i.e. $p_{\mathcal{A}} p=p_{\mathcal{A}}=p p_{\mathcal{A}}$. From $p \mathcal{A}=p \mathcal{B}, \mathcal{A} p=\mathcal{B} p$, we obtain $p_{\mathcal{A}} p \mathcal{A}=p_{\mathcal{A}} p \mathcal{B}$, $\mathcal{A} p p_{\mathcal{A}}=\mathcal{B}_{p} p_{\mathcal{A}}$, and hence $p_{\mathcal{A}} \mathcal{A}=p_{\mathcal{A}} \mathcal{B}, \mathcal{A} p_{\mathcal{A}}=\mathcal{B} p_{\mathcal{A}}$, i.e. $\mathcal{A} \leq \sharp \mathcal{B}$.

(iii) $\Rightarrow\left(\right.$ iv): Suppose there exists an idempotent $p \in \mathcal{R}$ such that $l_{\mathcal{R}}(p) \subseteq$ $l_{\mathcal{R}}(\mathcal{A}), r_{\mathcal{R}}(p) \subseteq r_{\mathcal{R}}(\mathcal{A}), p \mathcal{A}=p \mathcal{B}$, and $\mathcal{A} p=\mathcal{B} p$. Since then $(1-p) \mathcal{A}=$ $\mathcal{A}(1-p)=\{0\}$ it follows that $\mathcal{A}=p \mathcal{A}=\mathcal{A} p$ and thus $\mathcal{A}=p \mathcal{B}=\mathcal{B} p$.

(iv) $\Rightarrow$ (iii): Suppose $\mathcal{A}=p \mathcal{B}=\mathcal{B} p$ for some idempotent $p \in \mathcal{R}$. Then $(1-p) \mathcal{A}=\mathcal{A}(1-p)=\{0\}$ and thus $l_{\mathcal{R}}(p) \subseteq l_{\mathcal{R}}(\mathcal{A})$ and $r_{\mathcal{R}}(p) \subseteq r_{\mathcal{R}}(\mathcal{A})$. Also, clearly, $p \mathcal{A}=p \mathcal{B}$ and $\mathcal{A} p=\mathcal{B} p$.

\section{THE ONE-SIDED SHARP ORDERS}

The sharp partial order (1.1) is defined with two conditions. If one of these conditions is removed and perhaps some milder condition is added, does one still get a partial order? Following definition (1.1), the left-sharp and the right-sharp partial orders were introduced on $\mathcal{G}(\mathcal{R})$ in [16]. For $a, b \in \mathcal{G}(\mathcal{R})$ we write

and

$$
a \sharp \leq b \quad \text { if } \quad a^{\sharp} a=a^{\sharp} b \quad \text { and } \quad l_{\mathcal{R}}(b) \subseteq l_{\mathcal{R}}(a)
$$

$$
a \leq \sharp b \quad \text { if } \quad a a^{\sharp}=b a^{\sharp} \quad \text { and } \quad r_{\mathcal{R}}(b) \subseteq r_{\mathcal{R}}(a) .
$$


The relations $\sharp \leq$ and $\leq \sharp$ are called the left-sharp order and the right-sharp order on $\mathcal{G}(\mathcal{R})$, respectively. In [16], it was shown that these relations are partial orders when $\mathcal{R}$ is a ring with identity. It was also proved that for $a \in \mathcal{G}(\mathcal{R})$ and $b \in \mathcal{R}$ the following holds: $a^{\sharp} a=a^{\sharp} b$ if and only if $p_{\{a\}} a=p_{\{a\}} b$, and $a a^{\sharp}=b a^{\sharp}$ if and only if $a p_{\{a\}}=b p_{\{a\}}$ (here it turns out that $p_{\{a\}}=a a^{\sharp}$ (see [16, Lemma 2.1])). Following these observations, we now extend the concept of one-sided sharp orders to $\mathcal{J}_{\mathcal{R}}$.

Definition 3.1. Let $\mathcal{A} \in \mathcal{J}_{\mathcal{R}}$ and $\mathcal{B} \subseteq \mathcal{R}$.

(i) We write $\mathcal{A} \sharp \leq \mathcal{B}$ when $p_{\mathcal{A}} \mathcal{A}=p_{\mathcal{A}} \mathcal{B}$ and $l_{\mathcal{R}}(\mathcal{B}) \subseteq l_{\mathcal{R}}(\mathcal{A})$. We call the relation $\sharp \leq$ the left-sharp order on $\mathcal{J}_{\mathcal{R}}$.

(ii) We write $\mathcal{A} \leq \sharp \mathcal{B}$ when $\mathcal{A} p_{\mathcal{A}}=\mathcal{B}_{p_{\mathcal{A}}}$ and $r_{\mathcal{R}}(\mathcal{B}) \subseteq r_{\mathcal{R}}(\mathcal{A})$. We call the relation $\leq \sharp$ the right-sharp order on $\mathcal{J}_{\mathcal{R}}$.

We show that these relations are partial orders on $\mathcal{J}_{\mathcal{R}}$ when $\mathcal{R}$ is a ring with identity. First, let us present a lemma which can be proved in a similar way as Lemma 2.3 and hence we omitted its proof.

Lemma 3.2. Let $\mathcal{A} \in \mathcal{J}_{\mathcal{R}}$ and $\mathcal{B} \subseteq \mathcal{R}$. If $\mathcal{A} \sharp \leq \mathcal{B}$, then $r_{\mathcal{R}}(\mathcal{B}) \subseteq r_{\mathcal{R}}(\mathcal{A})$, and if $\mathcal{A} \leq \sharp \mathcal{B}$, then $l_{\mathcal{R}}(\mathcal{B}) \subseteq l_{\mathcal{R}}(\mathcal{A})$.

Theorem 3.3. The relations $\sharp \leq$ and $\leq \sharp$ introduced with Definition 3.1 are partial orders on $\mathcal{J}_{\mathcal{R}}$.

Proof. Let us show that the left-sharp order $\sharp \leq$ is a partial order on $\mathcal{J}_{\mathcal{R}}$. The proof that the right-sharp order $\leq \sharp$ is a partial order is similar and we omit it.

Reflexivity clearly holds.

Antisymmetry: Let $\mathcal{A}, \mathcal{B} \in \mathcal{J}_{\mathcal{R}}$ with $\mathcal{A} \sharp \leq \mathcal{B}$ and $\mathcal{B} \sharp \leq \mathcal{A}$. Then $\mathcal{A}=p_{\mathcal{A}} \mathcal{A}=$ $p_{\mathcal{A}} \mathcal{B}, \mathcal{B}=p_{\mathcal{B}} \mathcal{B}=p_{\mathcal{B}} \mathcal{A}$, and $l_{\mathcal{R}}(\mathcal{B})=l_{\mathcal{R}}(\mathcal{A})$. Since $\left(1-p_{\mathcal{B}}\right) \mathcal{B}=\{0\}$, we have $\left(1-p_{\mathcal{B}}\right) \mathcal{A}=\{0\}$ which implies $\mathcal{A}=p_{\mathcal{B}} \mathcal{A}$. It follows that $\mathcal{A}=p_{\mathcal{B}} \mathcal{A}=p_{\mathcal{B}} \mathcal{B}=\mathcal{B}$.

Transitivity: Let $\mathcal{A}, \mathcal{B} \in \mathcal{J}_{\mathcal{R}}$ and $\mathcal{C} \subseteq \mathcal{R}$ with $\mathcal{A} \sharp \leq \mathcal{B}$ and $\mathcal{B} \sharp \leq \mathcal{C}$. Then $p_{\mathcal{A}} \mathcal{A}=p_{\mathcal{A}} \mathcal{B}, l_{\mathcal{R}}(\mathcal{B}) \subseteq l_{\mathcal{R}}(\mathcal{A})$, and $p_{\mathcal{B}} \mathcal{B}=p_{\mathcal{B}} \mathcal{C}, l_{\mathcal{R}}(\mathcal{C}) \subseteq l_{\mathcal{R}}(\mathcal{B})$. It follows that $l_{\mathcal{R}}(\mathcal{C}) \subseteq l_{\mathcal{R}}(\mathcal{A})$. Also, by Lemma 3.2 , we have $r_{\mathcal{R}}\left(p_{\mathcal{B}}\right)=r_{\mathcal{R}}(\mathcal{B}) \subseteq r_{\mathcal{R}}(\mathcal{A})=$ $r_{\mathcal{R}}\left(p_{\mathcal{A}}\right)$. So, $p_{\mathcal{A}}\left(1-p_{\mathcal{B}}\right)=0$ and thus $p_{\mathcal{A}}=p_{\mathcal{A}} p_{\mathcal{B}}$. From $p_{\mathcal{B}} \mathcal{B}=p_{\mathcal{B}} \mathcal{C}$ we obtain $p_{\mathcal{A}} p_{\mathcal{B}} \mathcal{B}=p_{\mathcal{A}} p_{\mathcal{B}} \mathcal{C}$ and thus $p_{\mathcal{A}} \mathcal{A}=p_{\mathcal{A}} \mathcal{B}=p_{\mathcal{A}} \mathcal{C}$, i.e. $\mathcal{A} \sharp \leq \mathcal{C}$.

TheOrEm 3.4. Let $\mathcal{A} \in \mathcal{J}_{\mathcal{R}}$ and $\mathcal{B} \subseteq \mathcal{R}$. Then the following statements are equivalent.

(i) $p_{\mathcal{A}} \mathcal{A}=p_{\mathcal{A}} \mathcal{B}$.

(ii) There exists an idempotent $p \in \mathcal{R}$ such that $\mathcal{A}=p \mathcal{A} p=p \mathcal{B} p$ and $p \mathcal{B}(1-p)=\{0\}$.

(iii) There exists an idempotent $p \in \mathcal{R}$ such that $r_{\mathcal{R}}(p) \subseteq r_{\mathcal{R}}(\mathcal{A})$ and $p \mathcal{A}=$ $p \mathcal{B}$.

(iv) For every $a \in \mathcal{A}$ there exists $b \in \mathcal{B}$ such that $b-a \in r_{\mathcal{R}}(\mathcal{A})$ and for every $b \in \mathcal{B}$ there exists $a \in \mathcal{A}$ such that $b-a \in r_{\mathcal{R}}(\mathcal{A})$. 
Proof. Let $\mathcal{A} \in \mathcal{J}_{\mathcal{R}}$ and $\mathcal{B} \subseteq \mathcal{R}$.

(i) $\Rightarrow$ (ii): Suppose (i) holds. By Remark 2.1, $\mathcal{A}=p_{\mathcal{A}} \mathcal{A}=\mathcal{A} p_{\mathcal{A}}$ and therefore $\mathcal{A}=p_{\mathcal{A}} \mathcal{A} p_{\mathcal{A}}$. Since $p_{\mathcal{A}} \mathcal{A}=p_{\mathcal{A}} \mathcal{B}$, it follows that $\mathcal{A}=p_{\mathcal{A}} \mathcal{B}_{\mathcal{A}_{\mathcal{A}}}$. Also, since $p_{\mathcal{A}} \mathcal{B} p_{\mathcal{A}}=\mathcal{A}=p_{\mathcal{A}} \mathcal{B}$, it follows that $p_{\mathcal{A}} \mathcal{B}\left(1-p_{\mathcal{A}}\right)=\{0\}$.

(ii) $\Rightarrow($ iii): Since (ii) holds, $p \mathcal{B}(1-p)=\{0\}$ for some idempotent $p \in \mathcal{R}$ and thus $p \mathcal{B}=p \mathcal{B} p$. We also have $\mathcal{A}=p \mathcal{A} p=p \mathcal{B} p$. So, $p \mathcal{A}=\mathcal{A}$ and $\mathcal{A}=p \mathcal{B} p=p \mathcal{B}$, and therefore $p \mathcal{A}=p \mathcal{B}$. Also, $\mathcal{A}(1-p)=\{0\}$ and hence $r_{\mathcal{R}}(p) \subseteq r_{\mathcal{R}}(\mathcal{A})$.

(iii) $\Rightarrow$ (i): Since (iii) holds, it follows that $r_{\mathcal{R}}(p) \subseteq r_{\mathcal{R}}\left(p_{\mathcal{A}}\right)$ for some idempotent $p \in \mathcal{R}$ which yields $p_{\mathcal{A}}(1-p)=0$, i.e. $p_{\mathcal{A}}=p_{\mathcal{A}} p$. From $p \mathcal{A}=p \mathcal{B}$ we have $p_{\mathcal{A}} p \mathcal{A}=p_{\mathcal{A}} p \mathcal{B}$ and hence $p_{\mathcal{A}} \mathcal{A}=p_{\mathcal{A}} \mathcal{B}$.

(iv) $\Leftrightarrow\left(\right.$ i): Let $a$ be any element in $\mathcal{A}$ and recall that $r_{\mathcal{R}}(\mathcal{A})=r_{\mathcal{R}}\left(p_{\mathcal{A}}\right)$. Then there exists $b \in \mathcal{B}$ such that $b-a \in r_{\mathcal{R}}(\mathcal{A})$, i.e. $\mathcal{A}(b-a)=\{0\}$, if and only if $p_{\mathcal{A}}(b-a)=0$, i.e. $p_{\mathcal{A}} a=p_{\mathcal{A}} b$. So, for every $a \in \mathcal{A}$ there exists $b \in \mathcal{B}$ such that $b-a \in r_{\mathcal{R}}(\mathcal{A})$ if and only if $p_{\mathcal{A}} \mathcal{A} \subseteq p_{\mathcal{A}} \mathcal{B}$. We similarly prove that for every $b \in \mathcal{B}$ there exists $a \in \mathcal{A}$ such that $b-a \in r_{\mathcal{R}}(\mathcal{A})$ if and only if $p_{\mathcal{A}} \mathcal{B} \subseteq p_{\mathcal{A}} \mathcal{A}$.

REMARK 3.5. Note that if (i) holds, then in (ii) we can always take $p=p_{\mathcal{A}}$. However, (ii) is not restricted to $p_{\mathcal{A}}$. Say, if $\mathcal{A}=\left\{2 E_{11}\right\} \subseteq M_{3}(\mathbb{R})$ and $\mathcal{B}=\left\{2 E_{11}+3 E_{22}\right\} \subseteq M_{3}(\mathbb{R})$, we may take $p=E_{11}+E_{33} \in M_{3}(\mathbb{R})$. Here $E_{i j}, 1 \leq i, j, \leq n$ denotes the standard basis of $M_{n}(\mathbb{R})$.

The following theorem could be shown similarly.

Theorem 3.6. Let $\mathcal{A} \in \mathcal{J}_{\mathcal{R}}$ and $\mathcal{B} \subseteq \mathcal{R}$. Then the following statements are equivalent.

(i) $\mathcal{A} p_{\mathcal{A}}=\mathcal{B} p_{\mathcal{A}}$.

(ii) There exists an idempotent $p \in \mathcal{R}$ such that $\mathcal{A}=p \mathcal{A} p=p \mathcal{B} p$ and $(1-p) \mathcal{B} p=\{0\}$

(iii) There exists an idempotent $p \in \mathcal{R}$ such that $l_{\mathcal{R}}(p) \subseteq l_{\mathcal{R}}(\mathcal{A})$ and $\mathcal{A} p=$ $\mathcal{B} p$.

(iv) For every $a \in \mathcal{A}$ there exists $b \in \mathcal{B}$ such that $b-a \in l_{\mathcal{R}}(\mathcal{A})$ and for every $b \in \mathcal{B}$ there exists $a \in \mathcal{A}$ such that $b-a \in l_{\mathcal{R}}(\mathcal{A})$.

Note that the three statements of Theorem 3.4 combined with the condition on left annihilators, i.e. $l_{\mathcal{R}}(\mathcal{B}) \subseteq l_{\mathcal{R}}(\mathcal{A})$, represent three equivalent definitions of the left-sharp order on $\mathcal{J}_{\mathcal{R}}$. Similarly, the three statements of Theorem 3.6 combined with the condition on right annihilators, i.e. $r_{\mathcal{R}}(\mathcal{B}) \subseteq r_{\mathcal{R}}(\mathcal{A})$, represent three equivalent definitions of the right-sharp order on $\mathcal{J}_{\mathcal{R}}$.

In [16], it was proved that for $a \in \mathcal{G}(\mathcal{R})$ and $b \in \mathcal{R}$ the following holds: $p_{\{a\}} a=p_{\{a\}} b$ if and only if $a^{2}=a b$, and $a p_{\{a\}}=b p_{\{a\}}$ if and only if $a^{2}=b a$. Does an analogue of this result hold for $\mathcal{A} \in \mathcal{J}_{\mathcal{R}}$ and $\mathcal{B} \subseteq \mathcal{R}$ ?

Lemma 3.7. Let $\mathcal{A} \in \mathcal{J}_{\mathcal{R}}$ and $\mathcal{B} \subseteq \mathcal{R}$. If $p_{\mathcal{A}} \mathcal{A}=p_{\mathcal{A}} \mathcal{B}$, then $\mathcal{A}^{2}=\mathcal{A B}$, and if $\mathcal{A} p_{\mathcal{A}}=\mathcal{B} p_{\mathcal{A}}$, then $\mathcal{A}^{2}=\mathcal{B} \mathcal{A}$. 
Proof. Let $\mathcal{A} \in \mathcal{J}_{\mathcal{R}}$. By Remark 2.1 we have $\mathcal{A}=p_{\mathcal{A}} \mathcal{A}=\mathcal{A}_{\mathcal{A}}$. If $p_{\mathcal{A}} \mathcal{A}=p_{\mathcal{A}} \mathcal{B}$ for $\mathcal{B} \subseteq \mathcal{R}$, then $\mathcal{A B}=\mathcal{A} p_{\mathcal{A}} \mathcal{B}=\mathcal{A} p_{\mathcal{A}} \mathcal{A}=\mathcal{A}^{2}$. We similarly prove that $\mathcal{A} p_{\mathcal{A}}=\mathcal{B}_{p_{\mathcal{A}}}$, implies $\mathcal{A}^{2}=\mathcal{B} \mathcal{A}$.

The converse of Lemma 3.7 need not be true in general as the following example shows.

EXAMPLE 3.8. Let $\mathbb{Z}_{6}$ denote the ring of integers modulo 6 . Then $\mathbb{Z}_{6}$ is a commutative ring and all of the idempotents of $\mathbb{Z}_{6}$ are $\overline{0}, \overline{1}, \overline{3}$, and $\overline{4}$. Consider the subsets $\mathcal{A}=\{\overline{0}, \overline{3}\}$ and $\mathcal{B}=\{\overline{1}\}$ of $\mathbb{Z}_{6}$. We have $r_{\mathbb{Z}_{6}}(\mathcal{A})=r_{\mathbb{Z}_{6}}(\overline{3})=\overline{4}$ and $l_{\mathbb{Z}_{6}}(\mathcal{A})=l_{\mathbb{Z}_{6}}(\overline{3})=\overline{4}$, and thus $\mathcal{A} \in \mathcal{J}_{\mathbb{Z}_{6}}$ and $p_{\mathcal{A}}=\overline{3}$. On the one hand, $\mathcal{A}^{2}=\mathcal{A}=\mathcal{A B}$, but on the other hand, $p_{\mathcal{A}} \mathcal{A}=\mathcal{A}$ and $p_{\mathcal{A}} \mathcal{B}=\{\overline{3}\}$, and so $p_{\mathcal{A}} \mathcal{A} \neq p_{\mathcal{A}} \mathcal{B}$.

Rakić introduced another generalization of the left-sharp and the rightsharp orders in [22]. Namely, for $a, b \in \mathcal{R}$, we say that $a \sharp_{1} \leq b$ if $a \in \mathcal{I}_{\mathcal{R}}$ and $a=p_{\{a\}} b=b q$ for some idempotent $q \in \mathcal{R}$ with $r_{\mathcal{R}}(a)=r_{\mathcal{R}}(q)$. We write $a \leq \sharp_{1} b$ if $a \in \mathcal{I}_{\mathcal{R}}$ and $a=q b=b p_{\{a\}}$ for some idempotent $q \in \mathcal{R}$ with $l_{\mathcal{R}}(a)=l_{\mathcal{R}}(q)$. Following this approach, we now introduce two new relations on $\mathcal{J}_{\mathcal{R}}$ and then prove that they again represent partial orders on $\mathcal{J}_{\mathcal{R}}$.

Definition 3.9. Let $\mathcal{A} \in \mathcal{J}_{\mathcal{R}}$ and $\mathcal{B} \subseteq \mathcal{R}$.

(i) We write $\mathcal{A}_{1} \leq \mathcal{B}$ when there exists an idempotent $q$ with $r_{\mathcal{R}}(\mathcal{A})=$ $r_{\mathcal{R}}(q)$ and

$$
\mathcal{A}=p_{\mathcal{A}} \mathcal{B}=\mathcal{B} q
$$

(ii) We write $\mathcal{A} \leq \sharp_{1} \mathcal{B}$ when there exists an idempotent $q$ with $l_{\mathcal{R}}(\mathcal{A})=$ $l_{\mathcal{R}}(q)$ and

$$
\mathcal{A}=q \mathcal{B}=\mathcal{B} p_{\mathcal{A}}
$$

THEOREM 3.10. The relations $\sharp_{1} \leq$ and $\leq \sharp_{1}$ introduced with Definitions 3.9 are partial orders on $\mathcal{J}_{\mathcal{R}}$.

Proof. We show that $\sharp_{1} \leq$ is a partial order on $\mathcal{J}_{\mathcal{R}}$. The proof that $\leq \sharp_{1}$ is a partial order on $\mathcal{J}_{\mathcal{R}}$ is similar and so we omit it.

Reflexivity is clear since $\mathcal{A} \in \mathcal{J}_{\mathcal{R}}$ and thus $\mathcal{A}=p_{\mathcal{A}} \mathcal{A}=\mathcal{A} p_{\mathcal{A}}$.

Antisymmetry: Let $\mathcal{A}, \mathcal{B} \in \mathcal{J}_{\mathcal{R}}$ with $\mathcal{A} \sharp_{1} \leq \mathcal{B}$ and $\mathcal{B} \sharp_{1} \leq \mathcal{A}$. Then $\mathcal{A}=$ $p_{\mathcal{A}} \mathcal{B}=\mathcal{B} q$ for some idempotent $q \in \mathcal{R}$ with $r_{\mathcal{R}}(\mathcal{A})=r_{\mathcal{R}}(q)$, and $\mathcal{B}=p_{\mathcal{B}} \mathcal{A}=$ $\mathcal{A} r$ for some idempotent $r \in \mathcal{R}$ with $r_{\mathcal{R}}(\mathcal{B})=r_{\mathcal{R}}(r)$. Since $\mathcal{A}=\mathcal{B} q$ and $\mathcal{B}=\mathcal{A} r$, it follows that $l_{\mathcal{R}}(\mathcal{A})=l_{\mathcal{R}}(\mathcal{B})$ and therefore $l_{\mathcal{R}}(\mathcal{A})=l_{\mathcal{R}}\left(p_{\mathcal{B}}\right)$. So, $\left(1-p_{\mathcal{B}}\right) \mathcal{A}=\{0\}$ and therefore $\mathcal{A}=p_{\mathcal{B}} \mathcal{A}$. This yields

$$
\mathcal{A}=p_{\mathcal{B}} \mathcal{A}=\mathcal{B} \text {. }
$$

Transitivity: Let $\mathcal{A}, \mathcal{B} \in \mathcal{J}_{\mathcal{R}}$ and $\mathcal{C} \subseteq \mathcal{R}$ with $\mathcal{A} \sharp_{1} \leq \mathcal{B}$ and $\mathcal{B} \sharp_{1} \leq \mathcal{C}$. Then there exist idempotents $q, r \in \mathcal{R}$ such that $r_{\mathcal{R}}(\mathcal{A})=r_{\mathcal{R}}(q), r_{\mathcal{R}}(\mathcal{B})=r_{\mathcal{R}}(r)$, $\mathcal{A}=p_{\mathcal{A}} \mathcal{B}=\mathcal{B} q$, and $\mathcal{B}=p_{\mathcal{B}} \mathcal{C}=\mathcal{C} r$. Since $\mathcal{A}=p_{\mathcal{A}} \mathcal{B}$, we have $r_{\mathcal{R}}\left(p_{\mathcal{B}}\right)=$ 
PARTIAL ORDERS ON A CERTAIN SUBSET OF THE POWER SET OF RINGS 185

$r_{\mathcal{R}}(\mathcal{B}) \subseteq r_{\mathcal{R}}(\mathcal{A})=r_{\mathcal{R}}\left(p_{\mathcal{A}}\right)$ and therefore $p_{\mathcal{A}}\left(1-p_{\mathcal{B}}\right)=0$, i.e. $p_{\mathcal{A}}=p_{\mathcal{A}} p_{\mathcal{B}}$. It follows

$$
p_{\mathcal{A}} \mathcal{C}=p_{\mathcal{A}} p_{\mathcal{B}} \mathcal{C}=p_{\mathcal{A}} \mathcal{B}=\mathcal{A} .
$$

Let $s=r q$. We have

$$
\mathcal{C} s=\mathcal{C} r q=\mathcal{B} q=\mathcal{A} .
$$

To conclude the proof, we show that $s$ is an idempotent with $r_{\mathcal{R}}(s)=r_{\mathcal{R}}(\mathcal{A})$. Let $a \in \mathcal{A}$. Since $\mathcal{A}=p_{\mathcal{A}} \mathcal{B}$, there exists $b \in \mathcal{B}$ such that $a=p_{\mathcal{A}} b$. Since $r$ is an idempotent and $\mathcal{B}=\mathcal{C} r$, we may conclude that $b=b r$, and so

$$
a s=a r q=p_{\mathcal{A}} b r q=p_{\mathcal{A}} b q=a q .
$$

By $\mathcal{A}=\mathcal{B} q$ there exists $b_{1} \in \mathcal{B}$ such that $a=b_{1} q$. It follows that $a q=b_{1} q^{2}=$ $b_{1} q=a$. Hence $a s=a$ and thus $a(1-s)=0$ for every $a \in \mathcal{A}$. Therefore $1-s \in r_{\mathcal{R}}(\mathcal{A})=r_{\mathcal{R}}(q)$. It follows that $0=q(1-s)$ and hence $q=q s=q r q$. So, $s^{2}=r q r q=r q=s$. Moreover, since $s=r q$ and $q=q s$, we may conclude that $r_{\mathcal{R}}(s)=r_{\mathcal{R}}(q)=r_{\mathcal{R}}(\mathcal{A})$. It follows that $\mathcal{A}_{1} \leq \mathcal{C}$.

Let $\mathcal{A} \in \mathcal{J}_{\mathcal{R}}, \mathcal{B} \subseteq \mathcal{R}$, and $q \in \mathcal{R}$. If $\mathcal{A}=\mathcal{B} q$, then $l_{\mathcal{R}}(\mathcal{B}) \subseteq l_{\mathcal{R}}(\mathcal{A})$ and therefore we may conclude that $\mathcal{A} \sharp_{1} \leq \mathcal{B}$ implies $\mathcal{A} \sharp \leq \mathcal{B}$. Similarly, $\mathcal{A} \leq \sharp_{1} \mathcal{B}$ yields $\mathcal{A} \leq \sharp \mathcal{B}$. Under what conditions do the converses of these two statements hold? We leave this as an open question.

3.1. One-sided sharp orders for operators on Hilbert spaces. We now consider one-sided sharp orders of the ring $B(\mathcal{H})$ of all bounded linear operators on a Hilbert space $\mathcal{H}$. We begin with some auxiliary results. For a $*$-ring $\mathcal{R}$ and $\mathcal{A} \subseteq \mathcal{R}$, let $\mathcal{A}^{*}=\left\{a^{*} \in \mathcal{R}: a \in \mathcal{A}\right\}$.

Lemma 3.11. Let $\mathcal{R}$ be $a *$-ring and $\mathcal{A}, \mathcal{B} \subseteq \mathcal{R}$. Then $l_{\mathcal{R}}(\mathcal{A}) \subseteq l_{\mathcal{R}}(\mathcal{B})$ if and only if $r_{\mathcal{R}}\left(\mathcal{A}^{*}\right) \subseteq r_{\mathcal{R}}\left(\mathcal{B}^{*}\right)$.

Proof. Let $\mathcal{A}, \mathcal{B} \subseteq \mathcal{R}$ with $l_{\mathcal{R}}(\mathcal{A}) \subseteq l_{\mathcal{R}}(\mathcal{B})$. Suppose $z \in r_{\mathcal{R}}\left(\mathcal{A}^{*}\right)$. Then $a^{*} z=0$ and thus $z^{*} a=0$ for every $a \in \mathcal{A}$. So, $z^{*} \in l_{\mathcal{R}}(\mathcal{A}) \subseteq l_{\mathcal{R}}(\mathcal{B})$ which yields that $z^{*} b=0$ for every $b \in \mathcal{B}$. It follows that $b^{*} z=0$ for every $b \in \mathcal{B}$ and thus $z \in r_{\mathcal{R}}\left(\mathcal{B}^{*}\right)$, i.e. $r_{\mathcal{R}}\left(\mathcal{A}^{*}\right) \subseteq r_{\mathcal{R}}\left(\mathcal{B}^{*}\right)$. The converse implication can be proved similarly.

Lemma 3.12. Let $\mathcal{A}, \mathcal{B} \subseteq B(\mathcal{H})$. Then

$$
r_{B(\mathcal{H})}(\mathcal{A}) \subseteq r_{B(\mathcal{H})}(\mathcal{B}) \text { if and only if } \bigcap_{G \in \mathcal{A}} \operatorname{Ker} G \subseteq \bigcap_{F \in \mathcal{B}} \operatorname{Ker} F \text {. }
$$

Proof. Let $\mathcal{A}, \mathcal{B} \subseteq B(\mathcal{H})$ and let $r_{B(\mathcal{H})}(\mathcal{A}) \subseteq r_{B(\mathcal{H})}(\mathcal{B})$. Suppose $x \in$ $\bigcap_{G \in \mathcal{A}} \operatorname{Ker} G$. Since $\bigcap_{G \in \mathcal{A}} \operatorname{Ker} G$ is a closed subspace of $\mathcal{H}$, there exists a projection $P \in B(\mathcal{H})$ such that $\operatorname{Im} P=\bigcap_{G \in \mathcal{A}} \operatorname{Ker} G$. So, $P x=x$ and $G P=0$ for every $G \in \mathcal{A}$. It follows that $F P=0$ for every $F \in \mathcal{B}$ and therefore $0=F P x=F x$ for every $F \in \mathcal{B}$. Thus, $x \in \bigcap_{F \in \mathcal{B}} \operatorname{Ker} F$, i.e. $\bigcap_{G \in \mathcal{A}} \operatorname{Ker} G \subseteq \bigcap_{F \in \mathcal{B}} \operatorname{Ker} F$. 
Conversely, let $\bigcap_{G \in \mathcal{A}} \operatorname{Ker} G \subseteq \bigcap_{F \in \mathcal{B}} \operatorname{Ker} F$ and suppose $X \in r_{B(\mathcal{H})}(\mathcal{A})$. Then $G X=0$ for every $G \in \mathcal{A}$ which yields $\operatorname{Im} X \subseteq \operatorname{Ker} G$ for every $G \in \mathcal{A}$. So, $\operatorname{Im} X \subseteq \bigcap_{G \in \mathcal{A}} \operatorname{Ker} G$ and thus $\operatorname{Im} X \subseteq \bigcap_{F \in \mathcal{B}} \operatorname{Ker} F$. It follows that $F X=0$ for every $F \in \mathcal{B}$ and so $r_{B(\mathcal{H})}(\mathcal{A}) \subseteq r_{B(\mathcal{H})}(\mathcal{B})$.

Lemma 3.13. Let $\mathcal{A}, \mathcal{B} \subseteq B(\mathcal{H})$. Then

$$
l_{B(\mathcal{H})}(\mathcal{A}) \subseteq l_{B(\mathcal{H})}(\mathcal{B}) \quad \text { if and only if } \overline{\sum_{F \in \mathcal{B}} \overline{\operatorname{Im} F}} \subseteq \overline{\sum_{G \in \mathcal{A}} \overline{\operatorname{Im} G}} .
$$

Proof. Let $\mathcal{A}, \mathcal{B} \subseteq B(\mathcal{H})$. By Lemma 3.11, $l_{B(\mathcal{H})}(\mathcal{A}) \subseteq l_{B(\mathcal{H})}(\mathcal{B})$ if and only if $r_{B(\mathcal{H})}\left(\mathcal{A}^{*}\right) \subseteq r_{B(\mathcal{H})}\left(\mathcal{B}^{*}\right)$ which is by Lemma 3.12 equivalent to $\bigcap_{G \in \mathcal{A}^{*}} \operatorname{Ker} G \subseteq \bigcap_{F \in \mathcal{B}^{*}} \operatorname{Ker} F$ and this is further equivalent to $\bigcap_{A \in \mathcal{A}} \operatorname{Ker} A^{*} \subseteq \bigcap_{B \in \mathcal{B}} \operatorname{Ker} B^{*}$. It follows that $l_{B(\mathcal{H})}(\mathcal{A}) \subseteq l_{B(\mathcal{H})}(\mathcal{B})$ if and

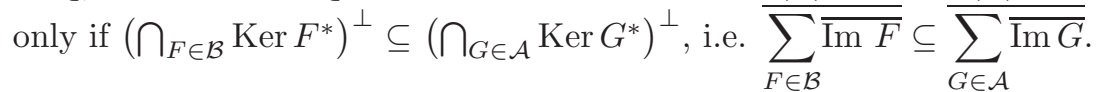

The following theorem is a direct consequence of Lemmas 3.12 and 3.13, and Definition 3.1.

Theorem 3.14. Let $\mathcal{A} \in \mathcal{J}_{B(\mathcal{H})}$ and $\mathcal{B} \subseteq B(\mathcal{H})$. Then

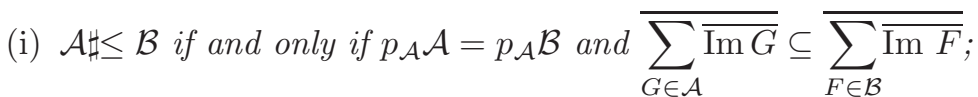

(ii) $\mathcal{A} \leq \sharp \mathcal{B}$ if and only if $\mathcal{A} p_{\mathcal{A}}=\mathcal{B} p_{\mathcal{A}}$ and $\bigcap_{F \in \mathcal{B}} \operatorname{Ker} F \subseteq \bigcap_{G \in \mathcal{A}} \operatorname{Ker} G$.

\section{ThE STAR, THE ONE-SIDED STAR, AND THE CORE ORDERS}

A $*$-ring $\mathcal{R}$ is called a Rickart $*$-ring if the left (equivalently, right) annihilator of any element $a \in \mathcal{R}$ is generated by a (unique) projection. Recall that a $*$-ring $\mathcal{R}$ is called proper if $a a^{*}=0$ implies $a=0$ for every $a \in \mathcal{R}$, and note that every Rickart $*$-ring is a proper $*$-ring [4]. In [8], Drazin introduced another partial order, known as the star partial order. Although introduced in a general setting of proper $*$-semigroups, this order was mostly studied on examples of proper $*$-rings, e.g. on the set of all $n \times n$ complex matrices, on $B(\mathcal{H})$, on Rickart $*$-rings (see [20], references therein, and $[1,7,17]$ ). Baksalary and Mitra introduced the left-star and the right-star partial orders in [2] on the set of all $m \times n$ complex matrices and Dolinar et al. generalized in [6] this concept to $B(\mathcal{H})$ by using idempotent operators. In [17], the left-star and the right-star partial orders were further generalized from $B(\mathcal{H})$ to Rickart *-rings. Very recently, in [24], these notions were extended to the power sets of Baer $*$-rings. The definitions are as follows.

Definition 4.1. Let $\mathcal{R}$ be $a *$-ring and $\mathcal{A}, \mathcal{B} \subseteq \mathcal{R}$. We write 
PARTIAL ORDERS ON A CERTAIN SUBSET OF THE POWER SET OF RINGS 187

(i) $\mathcal{A} \leq \mathcal{B}$ if there exist projections $p$ and $q$ in $\mathcal{R}$ such that $l_{\mathcal{R}}(\mathcal{A})=l_{\mathcal{R}}(p)$, $r_{\mathcal{R}}(\mathcal{A})=r_{\mathcal{R}}(q), p \mathcal{A}=p \mathcal{B}$, and $\mathcal{A} q=\mathcal{B} q$. We call the relation $\leq$ the star relation on $\mathcal{P}(\mathcal{R})$;

(ii) $\mathcal{A} * \leq \mathcal{B}$ if there exist a projection $p$ and an idempotent $q$ in $\mathcal{R}$ such that $l_{\mathcal{R}}(\mathcal{A})=l_{\mathcal{R}}(p), r_{\mathcal{R}}(\mathcal{A})=r_{\mathcal{R}}(q), p \mathcal{A}=p \mathcal{B}$, and $\mathcal{A} q=\mathcal{B} q$. We call the relation $* \leq$ the left-star relation on $\mathcal{P}(\mathcal{R})$;

(iii) $\mathcal{A} \leq * \mathcal{B}$ if there exist an idempotent $p$ and a projection $q$ in $\mathcal{R}$ such that $l_{\mathcal{R}}(\mathcal{A})=l_{\mathcal{R}}(p), r_{\mathcal{R}}(\mathcal{A})=r_{\mathcal{R}}(q), p \mathcal{A}=p \mathcal{B}$, and $\mathcal{A} q=\mathcal{B} q$. We call the relation $\leq *$ the right-star relation on $\mathcal{P}(\mathcal{R})$.

In [24], it was proved that the relations $\leq, * \leq$, and $\leq *$ are partial orders when $\mathcal{R}$ is a Baer $*$-ring. Moreover, in [24], it was also shown that the conditions in Definition 4.1 may be somewhat relaxed when $\mathcal{R}$ is a Baer *-ring. Namely, the following result was proved.

Proposition 4.2. Let $\mathcal{R}$ be a Baer $*$-ring and $\mathcal{A}, \mathcal{B} \subseteq \mathcal{R}$. Then the following statements hold:

(i) $\mathcal{A} \leq \mathcal{B}$ if and only if there exist projections $p, q \in \mathcal{R}$ such that $l_{\mathcal{R}}(p) \subseteq$ $l_{\mathcal{R}}(\mathcal{A}), r_{\mathcal{R}}(q) \subseteq r_{\mathcal{R}}(\mathcal{A}), p \mathcal{A}=p \mathcal{B}$, and $\mathcal{A} q=\mathcal{B} q$

(ii) $\mathcal{A} * \leq \mathcal{B}$ if and only if there exist a projection $p$ and an idempotent $q$ in $\mathcal{R}$ such that $l_{\mathcal{R}}(p) \subseteq l_{\mathcal{R}}(\mathcal{A}), r_{\mathcal{R}}(q) \subseteq r_{\mathcal{R}}(\mathcal{A}), p \mathcal{A}=p \mathcal{B}$, and $\mathcal{A} q=\mathcal{B} q$;

(iii) $\mathcal{A} \leq * \mathcal{B}$ if and only if there exist an idempotent $p$ and a projection $q$ in $\mathcal{R}$ such that $l_{\mathcal{R}}(p) \subseteq l_{\mathcal{R}}(\mathcal{A}), r_{\mathcal{R}}(q) \subseteq r_{\mathcal{R}}(\mathcal{A}), p \mathcal{A}=p \mathcal{B}$, and $\mathcal{A} q=\mathcal{B} q$.

Note that if $l_{\mathcal{R}}(s)=l_{\mathcal{R}}(t)$ for some projections $s, t \in \mathcal{R}$, then $(1-s) t=$ $0=(1-t) s$ and therefore $t=s t$ and $s=t s$. It follows that $s=s^{*}=s t=t$. For a Baer $*$-ring $\mathcal{R}$ and $\mathcal{A} \subseteq \mathcal{R}$ we may conclude the projection $p$ where $l_{\mathcal{R}}(\mathcal{A})=l_{\mathcal{R}}(p)$ is unique. We denote it by $\ln (\mathcal{A})$. Similarly, there exists the unique projection $r p(\mathcal{A})$ such that $r_{\mathcal{R}}(\mathcal{A})=r_{\mathcal{R}}(r p(\mathcal{A}))$. As a corollary to Proposition 4.2, we now present a new characterization of the star, the left-star, and the right-star partial orders on $\mathcal{P}(\mathcal{R})$ where $\mathcal{R}$ is a Baer $*$-ring.

Theorem 4.3. Let $\mathcal{R}$ be a Baer $*$-ring and $\mathcal{A}, \mathcal{B} \subseteq \mathcal{R}$. Then the following statements hold:

(i) $\mathcal{A} \leq \mathcal{B}$ if and only if $\mathcal{A}=\operatorname{lp}(\mathcal{A}) \mathcal{B}=\operatorname{Brp}(\mathcal{A})$;

(ii) $\mathcal{A} * \leq \mathcal{B}$ if and only if there exists an idempotent $q$ in $\mathcal{R}$ such that $\mathcal{A}=\operatorname{lp}(\mathcal{A}) \mathcal{B}=\mathcal{B} q$

(iii) $\mathcal{A} \leq * \mathcal{B}$ if and only if there exists an idempotent $p$ in $\mathcal{R}$ such that $\mathcal{A}=p \mathcal{B}=\mathcal{B} \operatorname{rp}(\mathcal{A})$.

Proof. Let $\mathcal{A} \leq \mathcal{B}$ for $\mathcal{A}, \mathcal{B} \subseteq \mathcal{R}$. By Definition $4.1, \operatorname{lp}(\mathcal{A}) \mathcal{A}=\operatorname{lp}(\mathcal{A}) \mathcal{B}$ and $\mathcal{A} r p(\mathcal{A})=\mathcal{B} r p(\mathcal{A})$. Since $l_{\mathcal{R}}(\mathcal{A})=l_{\mathcal{R}}(l p(\mathcal{A})), r_{\mathcal{R}}(\mathcal{A})=r_{\mathcal{R}}(r p(\mathcal{A}))$, we 
have $\mathcal{A}=\operatorname{lp}(\mathcal{A}) \mathcal{A}=\mathcal{A r p}(\mathcal{A})$ and therefore $\mathcal{A}=\operatorname{lp}(\mathcal{A}) \mathcal{B}=\operatorname{Brp}(\mathcal{A})$. Conversely, let $\mathcal{A}=\operatorname{lp}(\mathcal{A}) \mathcal{B}=\mathcal{B} r p(\mathcal{A})$. Then $\operatorname{lp}(\mathcal{A}) \mathcal{A}=\operatorname{lp}(\mathcal{A}) \mathcal{B}, \operatorname{Arp}(\mathcal{A})=$ $\mathcal{B} r p(\mathcal{A}), l_{\mathcal{R}}(l p(\mathcal{A})) \subseteq l_{\mathcal{R}}(\mathcal{A})$, and $r_{\mathcal{R}}(r p(\mathcal{A})) \subseteq r_{\mathcal{R}}(\mathcal{A})$, and hence by Proposition $4.2, \mathcal{A} \leq \mathcal{B}$.

Statements (ii) and (iii) can be proved similarly.

In [3], Baksalary and Trenkler introduced the core partial order on the set of all $n \times n$ complex matrices that have the group inverse (see also [13, 14]). This order was generalized by Rakić in [22] to $\mathcal{I}_{\mathcal{R}}$ where $\mathcal{R}$ is a Rickart $*$-ring. Namely, for $a, b \in \mathcal{R}$ we write

$$
a \leq{ }^{\oplus} b \quad \text { if } \quad a \in \mathcal{I}_{\mathcal{R}} \text { and } a=l p(a) b=b p_{\{a\}} .
$$

Motivated by this definition we extend the concept of the core order to $\mathcal{J}_{\mathcal{R}}$ and show that this relation is indeed a partial order when $\mathcal{R}$ is a Baer $*$-ring.

Definition 4.4. Let $\mathcal{R}$ be $a *$-ring, $\mathcal{A} \in \mathcal{J}_{\mathcal{R}}$ and $\mathcal{B} \subseteq \mathcal{R}$.

(i) We write

$$
\mathcal{A} \leq{ }^{\oplus} \mathcal{B} \quad \text { if } \quad \mathcal{A}=\operatorname{lp}(\mathcal{A}) \mathcal{B}=\mathcal{B} p_{\mathcal{A}} .
$$

We call the relation $\leq{ }^{\oplus}$ the core relation on $\mathcal{J}_{\mathcal{R}}$.

(ii) We write

$$
\mathcal{A} \leq_{\oplus} \mathcal{B} \quad \text { if } \quad \mathcal{A}=p_{\mathcal{A}} \mathcal{B}=\mathcal{B} r p(\mathcal{A})
$$

and we call the relation $\leq_{\oplus}$ the dual core relation on $\mathcal{J}_{\mathcal{R}}$.

The relations introduced with Definitions 4.4 are related to one-sided star and sharp orders as the next result shows.

Theorem 4.5. Let $\mathcal{R}$ be a Baer $*$-ring, $\mathcal{A} \in \mathcal{J}_{\mathcal{R}}$ and $\mathcal{B} \subseteq \mathcal{R}$. Then the following statements hold

(i) $\mathcal{A} \leq{ }^{\oplus} \mathcal{B}$ if and only if $\mathcal{A} * \leq \mathcal{B}$ and $\mathcal{A} \leq \sharp_{1} \mathcal{B}$;

(ii) $\mathcal{A} \leq_{\oplus} \mathcal{B}$ if and only if $\mathcal{A} \leq * \mathcal{B}$ and $\mathcal{A} \sharp_{1} \leq \mathcal{B}$.

Proof. Suppose $\mathcal{A} \leq{ }^{\boxplus} \mathcal{B}$, i.e. $\mathcal{A}=\operatorname{lp}(\mathcal{A}) \mathcal{B}=\mathcal{B} p_{\mathcal{A}}$. By Theorem 4.3 it follows that $\mathcal{A} * \leq \mathcal{B}$ and by Definition 3.9 (ii) we have $\mathcal{A} \leq \sharp_{1} \mathcal{B}$ since $l_{\mathcal{R}}(\mathcal{A})=l_{\mathcal{R}}(l p(\mathcal{A}))$. Conversely, if $\mathcal{A} * \leq \mathcal{B}$ and $\mathcal{A} \leq \sharp_{1} \mathcal{B}$, then by Theorem 4.3 and Definitions 3.9 (ii) and 4.4 (i), $\mathcal{A} \leq{ }^{\oplus} \mathcal{B}$.

The second statement can be proved similarly.

Since $* \leq, \leq \sharp_{1}, \leq *$, and $\sharp_{1} \leq$ are partial orders on $\mathcal{J}_{\mathcal{R}}$ when $\mathcal{R}$ is a Baer $*$-ring, we obtain the following result.

COROLlary 4.6. Let $\mathcal{R}$ be a Baer $*$-ring. The core relation $\leq^{\oplus}$ and the dual core relation $\leq_{\oplus}$ are partial orders on $\mathcal{J}_{\mathcal{R}}$. 
Let us conclude the paper with the following observation. Let $\mathcal{R}$ be a Baer ring and $\mathcal{A}, \mathcal{B} \subseteq \mathcal{R}$. In [24], it was shown that

$$
\mathcal{A} \leq^{-} \mathcal{B} \quad \text { if and only if } \quad \mathcal{A}=p \mathcal{B}=\mathcal{B} q
$$

for some idempotent elements $p, q \in \mathcal{R}$. Comparing (4.1) with Theorem 2.5 and Definitions 3.9 and 4.4 we may conclude that if for $\mathcal{A} \in \mathcal{J}_{\mathcal{R}}$ and $\mathcal{B} \subseteq \mathcal{R}$, $\mathcal{A} \leq \mathcal{B}$ where $\leq$ is one of the orders from the set $\left\{\leq^{\sharp}, \sharp_{1} \leq, \leq \sharp_{1}, \leq{ }^{\mathbb{B}}, \leq_{\mathbb{\oplus}}\right\}$, then $\mathcal{A} \leq^{-} \mathcal{B}$.

ACKNOWLEDGEMENTs.

The authors wish to thank the anonymous referees for helpful and constructive comments that improved the presentation of this paper.

\section{REFERENCES}

[1] J. Antezana, C. Cano, I. Mosconi and D. Stojanoff, A note on the star order in Hilbert spaces, Linear Multilinear Algebra 58 (2010), 1037-1051.

[2] J. K. Baksalary and S. K. Mitra, Left-star and right-star partial orderings, Linear Algebra Appl. 149 (1991), 73-89.

[3] O. M. Baksalary and G. Trenkler, Core inverse of matrices, Linear Multilinear Algebra 58 (2010), 681-697.

[4] S. K. Berberian, Baer *-rings, Springer-Verlag, New York, 1972.

[5] D. S. Djordjević, D. S. Rakić and J. Marovt, Minus partial order in Rickart rings, Publ. Math. Debrecen 87 (2015), 291-305.

[6] G. Dolinar, A. E. Guterman and J. Marovt, Monotone transformations on B(H) with respect to the left-star and the right-star partial order, Math. Inequal. Appl. 17 (2014), $573-589$.

[7] G. Dolinar and J. Marovt, Star partial order on B(H), Linear Algebra Appl. 434 (2011), 319-326.

[8] M. P. Drazin, Natural structures on semigroups with involution, Bull. Amer. Math. Soc. 84 (1978), 139-141.

[9] R. E. Hartwig, How to partially order regular elements, Math. Japon. 25 (1980), 1-13.

[10] R. E. Hartwig and J. Luh, A note on the group structure on unit regular ring elements, Pacific J. Math. 71 (1977), 449-461.

[11] A. Herrero and N. Thome, Sharp partial order and linear autonomous systems, Appl. Math. Comput. 366 (2020), 124736, 11 pp.

[12] I. Kaplansky, Rings of operators, Benjamin, New York, 1968.

[13] S. B. Malik, Some more properties of core partial order, Appl. Math. Comput. 221 (2013), 192-201.

[14] S. B. Malik, L. Rueda and N. Thome, Further properties on the core partial order and other matrix partial orders, Linear Multilinear Algebra 62 (2014), 1629-1648.

[15] J. Marovt, On partial orders in Rickart rings, Linear Multilinear Algebra 63 (2015), $1707-1723$.

[16] J. Marovt, One-sided sharp order in rings, J. Algebra Appl. 15 (2016), 1650161, 10 pp.

[17] J. Marovt, D. S. Rakić and D. S. Djordjević, Star, left-star, and right-star partial orders in Rickart *-rings, Linear Multilinear Algebra 63 (2015), 343-365.

[18] S. K. Mitra, On group inverses and the sharp order, Linear Algebra Appl. 92 (1987), 17-37.

[19] S. K. Mitra, Matrix partial order through generalized inverses: unified theory, Linear Algebra Appl. 148 (1991), 237-263. 
[20] S. K. Mitra, P. Bhimasankaram and S. B. Malik, Matrix partial orders, shorted operators and applications, World Scientific, Hackensack, 2010.

[21] M. Z. Nashed (ed.), Generalized inverses and applications, Academic Press, New YorkLondon, 1976.

[22] D. S. Rakić, Generalization of sharp and core partial order using annihilators, Banach J. Math. Anal. 9 (2015), 228-242.

[23] P. Šemrl, Automorphisms of $B(\mathcal{H})$ with respect to minus partial order, J. Math. Anal. Appl. 369 (2010), 205-213.

[24] B. Ungor, S. Halicioglu, A. Harmanci and J. Marovt, Partial orders on the power sets of Baer rings, J. Algebra Appl. 19 (2020), 2050011, 14 pp.

G. Dolinar

University of Ljubljana

Faculty of Electrical Engineering

Tržaška cesta 25, SI-1000 Ljubljana

Slovenia

and

IMFM

Jadranska ulica 19, SI-1000 Ljubljana

Slovenia

E-mail: gregor.dolinar@fe.uni-lj.si

B. Kuzma

University of Primorska

Glagoljaška 8, SI-6000 Koper

Slovenia

and

IMFM

Jadranska ulica 19, SI-1000 Ljubljana

Slovenia

E-mail: bojan.kuzma@upr.si

J. Marovt

University of Maribor

Faculty of Economics and Business

Razlagova 14, SI-2000 Maribor

Slovenia

and

IMFM

Jadranska ulica 19, SI-1000 Ljubljana

Slovenia

E-mail: janko.marovt@um.si

B. Ungor

Ankara University

Faculty of Sciences

Department of Mathematics

06100 Tandogan, Ankara

Turkey

E-mail: bungor@science.ankara.edu.tr

Received: 12.4.2019.

Revised: 11.11.2019. 